\title{
Relationships between Composition of Major Fatty Acids and Fat Distribution and Insulin Resistance in Japanese
}

\author{
Chikako Fujii, ${ }^{1}$ Toshihide Kawai, ${ }^{1,2,3}$ Koichiro Azuma, ${ }^{2}$ Yuko Oguma, ${ }^{2}$ \\ Fuminori Katsukawa, ${ }^{2}$ Hiroshi Hirose, ${ }^{1}$ Kumiko Tanaka, ${ }^{1}$ Shu Meguro, ${ }^{1}$ \\ Hideo Matsumoto, ${ }^{2}$ and Hiroshi Itoh ${ }^{1}$ \\ ${ }^{1}$ Department of Internal Medicine, Keio University School of Medicine, Tokyo, Japan \\ ${ }^{2}$ Institute for Integrated Sports Medicine, Keio University School of Medicine, Tokyo, Japan \\ ${ }^{3}$ Department of Internal Medicine, Tokyo Saiseikai Central Hospital, Tokyo, Japan \\ Correspondence should be addressed to Toshihide Kawai; tkawai@keio.jp
}

Received 5 January 2017; Revised 7 March 2017; Accepted 12 March 2017; Published 30 April 2017

Academic Editor: Joseph F. Ndisang

Copyright (c) 2017 Chikako Fujii et al. This is an open access article distributed under the Creative Commons Attribution License, which permits unrestricted use, distribution, and reproduction in any medium, provided the original work is properly cited.

\begin{abstract}
Objective. The aim of this study was to evaluate the relationships between the composition of free fatty acids (FFAs) and metabolic parameters, including body fat distribution, in Japanese. Methods. The study subjects were 111 Japanese patients (54 males, 57 females). Metabolic parameters and visceral and subcutaneous fat areas as determined by CT scanning at the umbilical level were measured. Glucose tolerance test (GTT) was performed by administering $75 \mathrm{~g}$ glucose orally. Results. The percentage of linoleic acid (C18:2), the greatest constituent among FFAs, was negatively correlated with visceral fat area $(r=-0.411, p<0.0001)$, fasting glucose $(r=-0.330, p<0.0001)$, HbA1c $(r=-0.231, p=0.0146)$, and systolic blood pressure $(r=-0.224, p=0.0184)$. Linoleic acid percentage was also significantly negatively correlated with HOMA-IR ( $r=-0.416, p<0.0001)$ by simple correlation. Based on the findings of OGTT, the 111 subjects were classified into three groups: 33 with normal glucose tolerance, 71 with impaired glucose tolerance (IGT), and 7 diabetic subjects. The percentage of serum linoleic acid in diabetic subjects was significantly lower than that in normal subjects. Conclusion. We conclude that serum linoleic acid level is negatively correlated with the accumulation of visceral fat in relation to a reduction of insulin resistance in Japanese subjects.
\end{abstract}

\section{Introduction}

Approximately 415 million people worldwide have diabetes mellitus [1], and the number is expected to increase to 642 million by the year $2040[1,2]$. Diabetes mellitus is associated with a markedly increased risk of coronary heart disease, stroke, and renal failure, as well as disability [3]. Free fatty acids (FFAs), which are absorbed from ingested food and also liberated by adipocytes and reassembled into triglycerides, are often elevated in obese individuals. The accumulation of visceral fat induces insulin resistance and worsens glucose and lipid metabolism and is important in the development of type 2 diabetes [4]. Recently, the effects of FFAs on insulin's action have been investigated intensively $[5,6]$. We have previously shown that FFAs cause $\beta$ cell damage mainly by apoptosis in vitro [7] and by ER stress in mice [8]. The serum composition of FFAs in humans differs from race to race, and from environment to environment, because it reflects the average fat intake in the preceding days [9-12]. Despite intensive in vitro and in vivo studies to elucidate the impairment of insulin's action induced by chronic elevation of FFAs, however, the physiological interactions whereby the chronic effects of fatty acids on pancreatic beta cells affect insulin's action on glucose and insulin resistance remain unclear. To clarify these issues, firstly we evaluated the relationships between serum FFA composition and body fat area as determined by computed tomographic (CT) scanning at the umbilical level. Second, we evaluated the relationships between three major FFAs and the presence of diabetes, in humans. 
TABLE 1: Baseline characteristics of subjects.

\begin{tabular}{|c|c|}
\hline Parameters & Values \\
\hline$N$ (male/female) & $111(54 / 57)$ \\
\hline Age (years) & $51.5 \pm 1.1$ \\
\hline Height $(\mathrm{cm})$ & $162.5 \pm 0.8$ \\
\hline Body mass index $\left(\mathrm{kg} / \mathrm{m}^{2}\right)$ & $26.4 \pm 0.5$ \\
\hline Waist circumference $(\mathrm{cm})$ & $91.3 \pm 1.4$ \\
\hline Systolic blood pressure $(\mathrm{mmHg})$ & $128.4 \pm 2.3$ \\
\hline Diastolic blood pressure $(\mathrm{mmHg})$ & $78.2 \pm 1.5$ \\
\hline \multicolumn{2}{|l|}{ Biochemical markers } \\
\hline Fasting plasma glucose (mg/dL) & $107.0 \pm 1.5$ \\
\hline Hemoglobin A1c (\%) & $5.7 \pm 0.9$ \\
\hline Immunoreactive insulin $(\mu \mathrm{U} / \mathrm{mL})$ & $9.4 \pm 0.7$ \\
\hline Total cholesterol (mg/dL) & $211.0 \pm 5.9$ \\
\hline High-density lipoprotein cholesterol (mg/dL) & $57.1 \pm 1.8$ \\
\hline Low-density lipoprotein cholesterol (mg/dL) & $123.8 \pm 4.4$ \\
\hline Triglyceride (mg/dL) & $131.4 \pm 8.0$ \\
\hline Free fatty acids $(\mathrm{mEq} / \mathrm{L})$ & $0.6 \pm 0.0$ \\
\hline Aspartate aminotransferase (IU/L) & $26.2 \pm 1.1$ \\
\hline Alanine aminotransferase (IU/L) & $31.9 \pm 2.5$ \\
\hline Creatinine $(\mathrm{mg} / \mathrm{dL})$ & $0.7 \pm 0.0$ \\
\hline Uric acid (mg/dL) & $5.8 \pm 0.1$ \\
\hline $\begin{array}{l}\text { High molecular weight adiponectin } \\
(\mu \mathrm{g} / \mathrm{mL})(n=63)\end{array}$ & $2.9 \pm 0.2$ \\
\hline Leptin $(\mathrm{ng} / \mathrm{mL})(n=63)$ & $12.4 \pm 1.5$ \\
\hline \multicolumn{2}{|l|}{$75 \mathrm{~g}$ OGTT } \\
\hline $30 \mathrm{~min}$ glucose $(\mathrm{mg} / \mathrm{dL})$ & $180.1 \pm 3.4$ \\
\hline $60 \mathrm{~min}$ glucose $(\mathrm{mg} / \mathrm{dL})$ & $199.9 \pm 5.3$ \\
\hline $120 \mathrm{~min}$ glucose $(\mathrm{mg} / \mathrm{dL})$ & $161.9 \pm 5.1$ \\
\hline $30 \mathrm{~min}$ IRI $(\mu \mathrm{U} / \mathrm{mL})$ & $50.4 \pm 3.7$ \\
\hline $60 \min$ IRI $(\mu \mathrm{U} / \mathrm{mL})$ & $70.1 \pm 5.3$ \\
\hline $120 \min$ IRI $(\mu \mathrm{U} / \mathrm{mL})$ & $68.7 \pm 4.7$ \\
\hline HOMA-IR & $2.4 \pm 0.2$ \\
\hline Insulinogenic index & $0.7 \pm 0.1$ \\
\hline \multicolumn{2}{|l|}{ CT scan } \\
\hline Visceral fat $\left(\mathrm{cm}^{2}\right)$ & $122.6 \pm 5.4$ \\
\hline Subcutaneous fat $\left(\mathrm{cm}^{2}\right)$ & $209.0 \pm 12.9$ \\
\hline $\mathrm{V} / \mathrm{S}$ ratio & $0.7 \pm 0.0$ \\
\hline
\end{tabular}

Data are mean \pm SEM. IRI: immunoreactive insulin; HOMA-IR: homeostasis model assessment insulin resistance index.

\section{Materials and Methods}

This cross-sectional study was conducted in accordance with the Declaration of Helsinki and was approved by the Ethics Review Board of Keio University.

2.1. Participants. We enrolled 111 Japanese patients (54 men and 57 women) who attended our outpatient sports clinic at Keio University Hospital in Tokyo (Table 1). In men, mean \pm standard error of the mean (SEM) age was $50.1 \pm 1.4$ years; body mass index (BMI), $26.7 \pm 0.7 \mathrm{~kg} / \mathrm{m}^{2}$;
TABLE 2: Component ratio analysis of twenty-four FFA fractions.

\begin{tabular}{lcc}
\hline Free fatty acid & Symbol & $\%$ \\
\hline Lauric acid & C12:0 & $0.098 \pm 0.91$ \\
Myristic acid & C14:0 & $1.04 \pm 0.40$ \\
Myristoleic acid & C14:1 & $0.098 \pm 0.04$ \\
Palmitic acid & C16:0 & $22.37 \pm 0.23$ \\
Palmitoleic acid & C16:1 & $2.57 \pm 0.10$ \\
Stearic acid & C18:0 & $6.86 \pm 0.10$ \\
Oleic acid & C18:1 & $20.06 \pm 0.32$ \\
Linoleic acid & C18:2 & $29.73 \pm 0.45$ \\
Gamma-linolenic acid & C18:3(6) & $0.31 \pm 0.16$ \\
Linolenic acid & C18:3(3) & $0.88 \pm 0.26$ \\
Arachidic acid & C20:0 & $0.051 \pm 0.002$ \\
Eicosenoic acid & C20:1 & $0.19 \pm 0.01$ \\
Eicosadienoic acid & C20:2 & $0.19 \pm 0.01$ \\
5-8-11-Eicosatrienoic acid & C20:3(9) & $0.054 \pm 0.003$ \\
Dihomo-gamma-linolenic acid & C20:3(6) & $1.19 \pm 0.03$ \\
Arachidonic acid & C20:4 & $5.64 \pm 0.13$ \\
Eicosapentaenoic acid & C20:5 & $2.87 \pm 0.19$ \\
Behenic acid & C22:0 & $0.11 \pm 0.01$ \\
Erucic acid & C22:1 & $0.026 \pm 0.003$ \\
Docosatetraenoic acid & C22:4 & $0.13 \pm 0.01$ \\
Docosapentaenoic acid & C22:5 & $0.82 \pm 0.03$ \\
Lignoceric acid & C24:0 & $0.044 \pm 0.003$ \\
Docosahexaenoic acid & C22:6 & $4.61 \pm 0.17$ \\
Nervonic acid & C24:1 & $0.11 \pm 0.05$ \\
\hline Data & & \\
Did & &
\end{tabular}

Data are mean \pm SEM

fasting plasma glucose (FPG), $109.2 \pm 2.4 \mathrm{mg} / \mathrm{dL}$; and hemoglobin $\mathrm{Alc}(\mathrm{HbAlc}), 5.6 \pm 0.2 \%$. In women, mean $\pm \mathrm{SEM}$ age was $52.7 \pm 1.8$ years; BMI, $26.2 \pm 0.8 \mathrm{~kg} / \mathrm{m}^{2}$; FPG, $104.9 \pm 2.0 \mathrm{mg} / \mathrm{dL}$; and $\mathrm{HbAlc}, 5.9 \pm 0.1 \%$. All subjects received dietary instructions for using a meal-exchange plan by nutritionists. The ideal dietary caloric intake for each patient was calculated as the ideal body weight (kilograms $) \times 25-30 \mathrm{kcal} / \mathrm{kg}$. We recommended them to take $50-60 \%$ total calories as carbohydrate, $20-25 \%$ as fat, and $15-20 \%$ as protein. The subjects' meal preference did not deviate from that of the standard Japanese population. The physical activity level for each subject was determined by a questionnaire. None of the subjects was taking any medication or was a smoker. The nature of the procedure was explained to the subjects, and written informed consent was obtained from all participants.

2.2. Measurements and OGTT. The body weight of each patient was measured at the sports clinic. BMI was determined as weight corrected for height: weight $(\mathrm{kg}) / \mathrm{height}$ $\left(\mathrm{m}^{2}\right)$. Blood pressure (BP) was determined in the sitting position after a 10-minute rest.

A fasting blood sample was drawn from each subject before breakfast in the early morning, after an overnight fast. Glucose tolerance test (GTT) was performed by administering $75 \mathrm{~g}$ glucose orally, and the results were classified as 
TABLE 3: Stepwise regression analysis of relationships between dependent variables and the most significant independent FFA variables.

\begin{tabular}{|c|c|c|c|c|}
\hline Dependent variable & Contributory cause & Correlation coefficient & $F$ value & $p$ value \\
\hline BMI $\left(\mathrm{kg} / \mathrm{m}^{2}\right)$ & Palmitic acid & $r=0.304$ & 14.707 & $<0.0001$ \\
\hline sBP (mmHg) & Linoleic acid & $r=-0.224$ & 5.732 & 0.0184 \\
\hline $\mathrm{dBP}(\mathrm{mmHg})$ & - & - & - & - \\
\hline \multicolumn{5}{|l|}{ Biomarkers } \\
\hline 0 min glu $(\mathrm{mg} / \mathrm{dL})$ & Linoleic acid & $r=-0.330$ & 9.383 & $<0.0001$ \\
\hline $120 \mathrm{~min}$ glu $(\mathrm{mg} / \mathrm{dL})$ & Linoleic acid & $r=-0.389$ & 14.041 & $<0.0001$ \\
\hline $0 \min \mathrm{IRI}(\mu \mathrm{U} / \mathrm{mL})$ & Myristic acid & $r=0.430$ & 14.014 & $<0.0001$ \\
\hline $120 \min$ IRI $(\mu \mathrm{U} / \mathrm{mL})$ & Palmitoleic acid & $r=0.342$ & 12.237 & 0.0006 \\
\hline $\operatorname{HbA1c}(N)(\%)$ & Linoleic acid & $r=-0.231$ & 6.165 & 0.0146 \\
\hline HOMA-IR & Myristic acid & $r=0.520$ & 13.792 & $<0.0001$ \\
\hline I.I. & - & - & - & - \\
\hline $\mathrm{TC}(\mathrm{mg} / \mathrm{dL})$ & Linolenic acid & $r=0.282$ & 5.554 & 0.0216 \\
\hline TG (mg/dL) & Oleic acid & $r=0.510$ & 26.853 & $<0.0001$ \\
\hline HDL-C (mg/dL) & Oleic acid & $r=-0.470$ & 18.722 & $<0.0001$ \\
\hline LDL-C (mg/dL) & Behenic acid & $r=-0.344$ & 6.584 & 0.0134 \\
\hline AST (IU/L) & Myristic acid & $r=0.270$ & 8.467 & 0.0044 \\
\hline ALT (IU/L) & Palmitic acid & $r=0.289$ & 9.86 & 0.0022 \\
\hline Cre $(\mathrm{mg} / \mathrm{dL})$ & Gamma linolenic acid & $r=-0.215$ & 6.022 & 0.0337 \\
\hline $\mathrm{UA}(\mathrm{mg} / \mathrm{dL})$ & Eicosenoic acid & $r=0.247$ & 6.002 & 0.0162 \\
\hline HMW-ADPN $(\mu \mathrm{g} / \mathrm{mL})(n=63)$ & Linoleic acid & $r=0.327$ & 12.917 & 0.0108 \\
\hline Leptin $(\mathrm{ng} / \mathrm{mL})(n=63)$ & Lauric acid & $r=0.303$ & 6.157 & 0.0159 \\
\hline \multicolumn{5}{|l|}{ CT scan } \\
\hline $\mathrm{V}$-fat $\left(\mathrm{cm}^{2}\right)$ & Linoleic acid & $r=-0.411$ & 17.73 & $<0.0001$ \\
\hline S-fat $\left(\mathrm{cm}^{2}\right)$ & Dihomo- $\gamma$-linolenic acid & $r=0.272$ & 9.221 & 0.0002 \\
\hline
\end{tabular}

BMI: body mass index; sBP: systolic blood pressure; dBP: diastolic blood pressure; 0 min glu: 0 min glucose; 120 min glu: 120 min glucose; 0 min IRI: 0 min immunoreactive insulin; 120 min IRI: 120 min-immunoreactive insulin; HbAlc: hemoglobin A1c; HOMA-IR: homeostasis model assessment insulin resistance index; I.I.: insulinogenic index; TC: total cholesterol; TG: triglyceride; HDL-C: high-density lipoprotein cholesterol; LDL-C: low-density lipoprotein cholesterol; AST: aspartate aminotransferase; ALT: alanine aminotransferase; Cre: creatinine; UA: uric acid; HMW-ADPN: high molecular weight adiponectin; V-fat: visceral fat area; S-fat: subcutaneous fat area.

normal glucose tolerance, impaired glucose tolerance (IGT), or diabetes on the basis of the World Health Organization criteria [13]. Plasma glucose (PG) was determined by the glucose oxidase method. Plasma immunoreactive insulin (IRI) level was measured by radioimmunoassay (Shionogi, Tokyo, Japan). Twenty-four fractions of FFAs (lauric, myristic, myristoleic, palmitic, palmitoleic, stearic, oleic, linoleic, gamma-linolenic, linolenic, arachidic, eicosenoic, eicosadienoic, 5-8-11-eicosatrienoic, dihomo-gamma-linolenic, arachidonic, eicosapentaenoic, behenic, erucic, docosatetraenoic, docosapentaenoic, lignoceric, docosahexaenoic, and nervonic acid) (Table 2) were determined by capillary gas chromatography (HP 6890, Hitachi, 30 m capillary column, inner diameter $0.32 \mathrm{~mm}$, phase layer $0.25 \mu \mathrm{m}$ ) [14]. The n6/n3 polyunsaturated fatty acid (PUFA) ratio was calculated as (linoleic + gamma linolenic + eicosadienoic + dihomo-gamma-linolenic + arachidonic + docosatetraenoic acid) / (linolenic + eicosapentaenoic + docosapentaenoic + docosahexaenoic acids). Glycosylated hemoglobin (HbAlc) was determined by high-performance liquid chromatography (HPLC) (Toso Co. Ltd., Tokyo, Japan). Total cholesterol (TC), high-density lipoprotein cholesterol (HDL-C), low-density lipoprotein cholesterol (LDL-C), and triglyceride (TG) were measured enzymatically (Hitachi autoanalyzer). Insulin resistance was assessed using the homeostasis model assessment (HOMA) system described by Matthews et al. [15] with the formula (insulin $(\mu \mathrm{U} / \mathrm{mL}) \times$ plasma glucose $(\mathrm{mg} / \mathrm{dL}) / 405)$. High molecular weight (HMW) adiponectin was determined by sensitive latex particle-enhanced turbidimetric immunoassay (LTIA) (Hitachi H7170, Japan). Leptin was determined by radioimmunoassay (Hitachi ARC-950, Japan).

2.3. Computed Tomography. Subcutaneous and visceral fat distributions were determined by measuring a -150 to -50 Hounsfield unit (HU) area using CT scanning at the umbilical level as described previously [16]. V/S ratio was calculated as visceral fat area/subcutaneous fat area.

2.4. Statistical Analysis. All results are expressed as mean \pm SEM. Relationships between variables were analyzed by simple correlation and by linear stepwise regression analysis with calculation of Pearson product correlation coefficients. The Kruskal-Wallis test was used to evaluate 


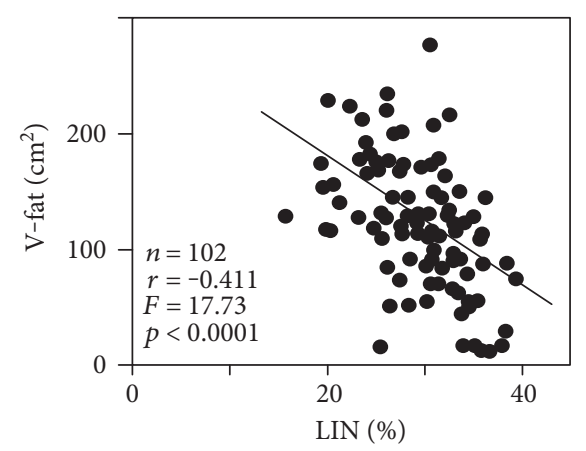

(a)

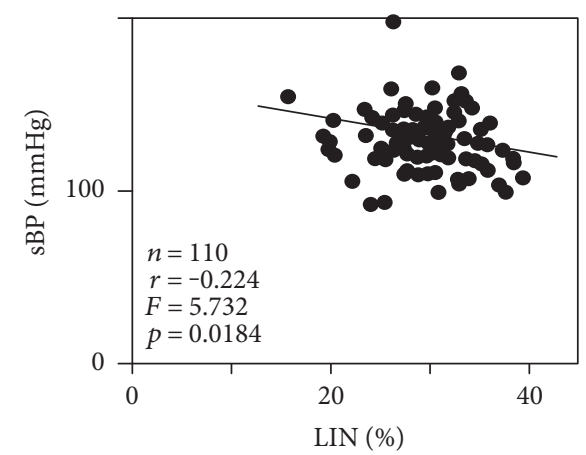

(c)

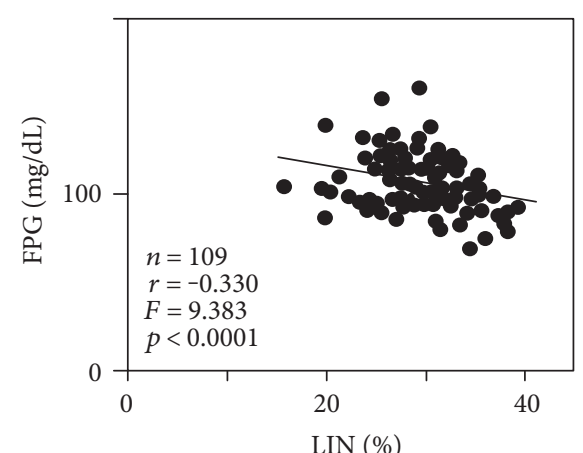

(b)

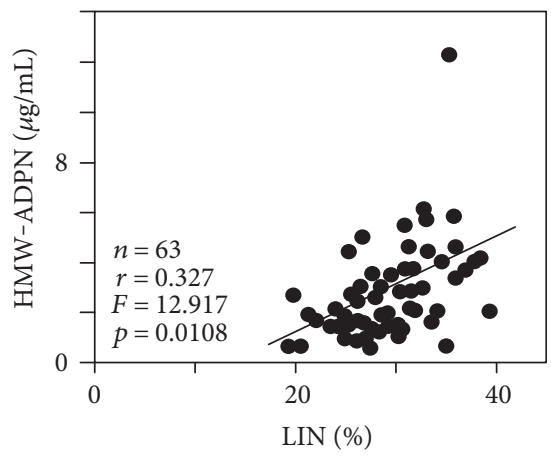

(d)

Figure 1: Relationships between ratio of serum linoleic acid (LIN, C18:2) and that of visceral fat area (V-fat) as determined by computed tomographic (CT) scanning at the umbilical level (a), that of fasting plasma glucose (FPG) (b), that of systolic blood pressure (sBP) (c), and that of high molecular weight (HMW) adiponectin (ADPN) (d) by linear stepwise regression analysis.

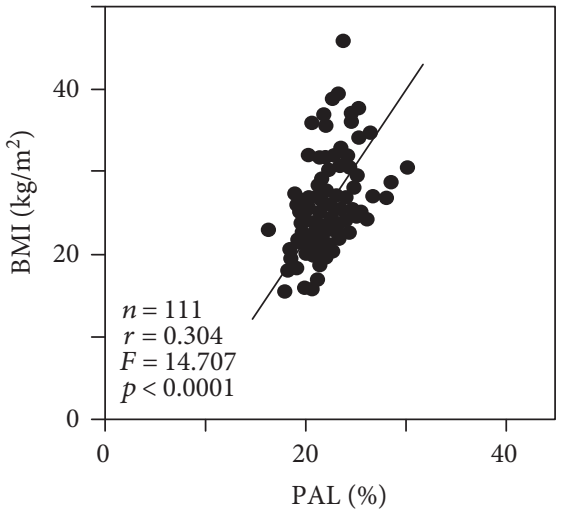

(a)

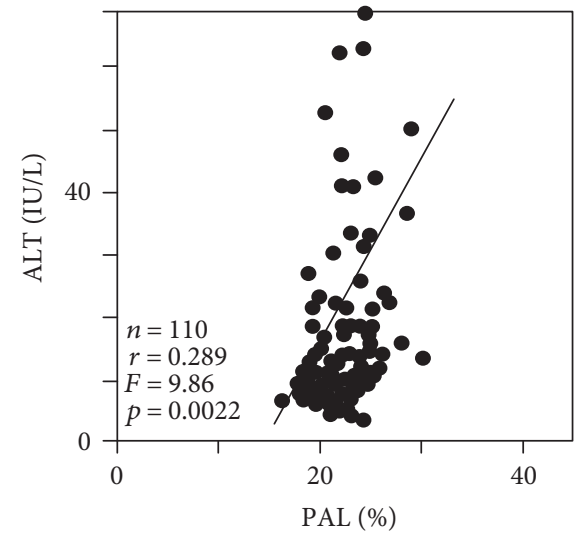

(b)

Figure 2: Relationships between ratio of serum palmitic acid (PAL, C16:0) and that of body mass index (BMI) (a) and that of alanine aminotransferase (ALT) (b).

comparisons among groups. A $p$ value less than 0.05 was considered statistically significant. Statistical analyses were carried out using SPSS Statistics 23 software (IBM, Armonk, NY, USA).

\section{Results}

3.1. Relationships between FFA Composition and Metabolic Parameters. We measured twenty-four fractions of FFAs in 


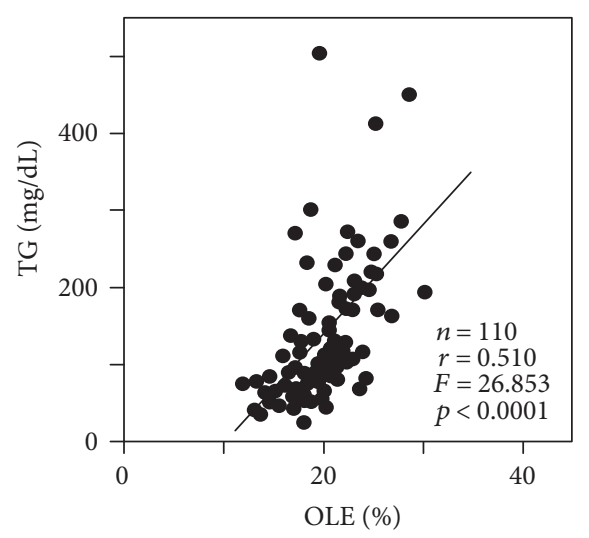

(a)

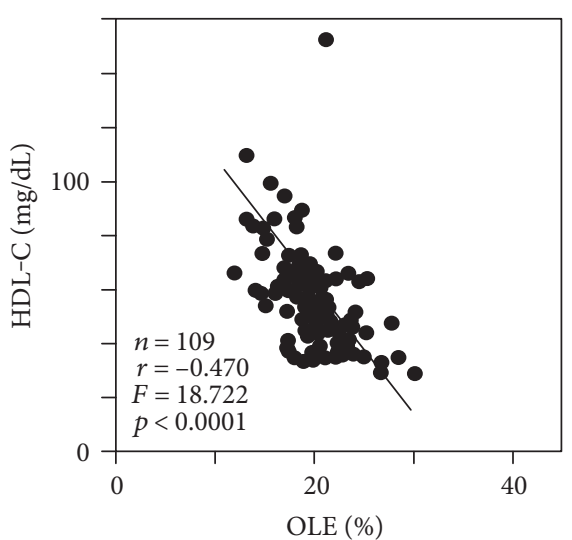

(b)

FIGURE 3: Relationships between ratio of serum oleic acid (OLE, C18:1) and that of triglyceride (TG) (a) and that of high-density lipoprotein cholesterol (HDL-C) (b).

TABLE 4: Subjects classified as normal glucose tolerant, impaired glucose tolerant (IGT), and diabetic based on OGTT.

\begin{tabular}{|c|c|c|c|c|}
\hline Parameters & Normal & IGT & Diabetes & $p$ value \\
\hline$N$ & 33 & 71 & 7 & - \\
\hline (Male/female) & $(13 / 20)$ & $(43 / 28)$ & $(2 / 5)$ & - \\
\hline Age (years) & $47.9 \pm 2.2$ & $52.5 \pm 1.4$ & $58.4 \pm 3.6$ & 0.0601 \\
\hline Body mass index $\left(\mathrm{kg} / \mathrm{m}^{2}\right)$ & $26.2 \pm 1.1$ & $26.5 \pm 0.7$ & $26.4 \pm 0.9$ & 0.808 \\
\hline Fasting glucose $(\mathrm{mg} / \mathrm{dL})$ & $93.8 \pm 1.6$ & $109.4 \pm 1.5$ & $141.6 \pm 4.8$ & $<0.0001$ \\
\hline Hemoglobin Alc (\%) & $5.2 \pm 0.2$ & $6.0 \pm 0.1$ & $6.4 \pm 0.1$ & $<0.0001$ \\
\hline HOMA-IR & $1.5 \pm 0.2$ & $2.7 \pm 0.3$ & $4.1 \pm 1.5$ & 0.0011 \\
\hline Visceral fat area $\left(\mathrm{cm}^{2}\right)$ & $115.6 \pm 11.5$ & $124.0 \pm 6.3$ & $141.3 \pm 17.3$ & 0.712 \\
\hline
\end{tabular}

Kruskal-Wallis test was used for comparisons among groups. Data are mean \pm SEM. HOMA-IR: homeostasis model assessment insulin resistance index.

all subjects, as shown in Table 2. Mean \pm SEM proportions of constituents were $29.7 \pm 0.5 \%$ linoleic acid, $22.4 \pm 0.2 \%$ palmitic acid, $20.1 \pm 0.3 \%$ oleic acid, $6.9 \pm 0.1 \%$ stearic acid, $5.6 \pm 0.1 \%$ arachidonic acid, and $4.6 \pm 0.2 \%$ docosahexaenoic acid, and the others were below $3.0 \%$ each. First, we analyzed the relationships between twenty-four FFA fractions and twenty-two metabolic parameters (BMI, sBP, dBP, 0 min glucose, $120 \mathrm{~min}$ glucose, $0 \mathrm{~min}$ IRI, $120 \mathrm{~min}$ IRI, HbA1c, HOMA-IR, I.I, TC, TG, HDL-C, LDL-C, aspartate aminotransferase (AST), alanine aminotransferase (ALT), creatinine, uric acid, HMW adiponectin, leptin, visceral fat area, and subcutaneous fat area) by simple correlation analysis (Supplementary Table available online at https:// doi.org/10.1155/2017/1567467). Second, multivariate analysis models were developed by stepwise regression with twenty-two metabolic parameters as dependent variables and by simple correlation with significant fractions among all twenty-four FFA fractions as independent variables. Table 3 shows the relationships between dependent metabolic variables and the most significant independent FFA variables.

As the most significant contributory cause, linoleic acid (\%), the greatest constituent among the twenty-four FFA fractions, was negatively correlated with visceral fat area $(r=-0.411, F=17.73, p<0.0001$ ) (Figure 1(a)), whereas it was not correlated with subcutaneous fat area. Linoleic acid was negatively correlated with FPG $(r=-0.330, F=9.383$, $p<0.0001)$ (Figure 1(b)) and $\operatorname{sBP}(r=-0.224, F=5.732$, $p=0.0184$ ) (Figure $1(\mathrm{c})$ ) and positively correlated with HMW adiponectin $(n=63, \quad r=0.327, \quad F=12.917, \quad p=$ 0.0108 ) (Figure $1(\mathrm{~d})$ ). In addition, linoleic acid was also significantly negatively correlated with $120 \mathrm{~min}$ IRI $(r=$ $-0.383, p<0.0001)$ and HOMA-IR $(r=-0.416, p<0.0001)$ by simple correlation analysis (Supplementary Table). Palmitic acid (\%) was positively correlated with BMI $(r=0.304, F=14.707, p<0.0001)$ (Figure $2(\mathrm{a}))$ and ALT $(r=0.289, F=9.86, p=0.0022)$ (Figure 2(b)). Oleic acid (\%) was positively correlated with TG $(r=0.510, F=26.853$, $p<0.0001$ ) (Figure 3(a)) and negatively correlated with HDL-C $(r=-0.470, F=18.722, p<0.0001)$ (Figure 3(b)).

3.2. OGTT and Fraction of Three Major FFAs. Based on the findings of OGTT, the 111 subjects were classified into three groups: 33 with normal glucose tolerance, 71 with IGT, and 7 diabetic subjects (Table 4). There were no significant differences in macronutrient balance and physical activity level among the three groups. Figure 4 shows the 


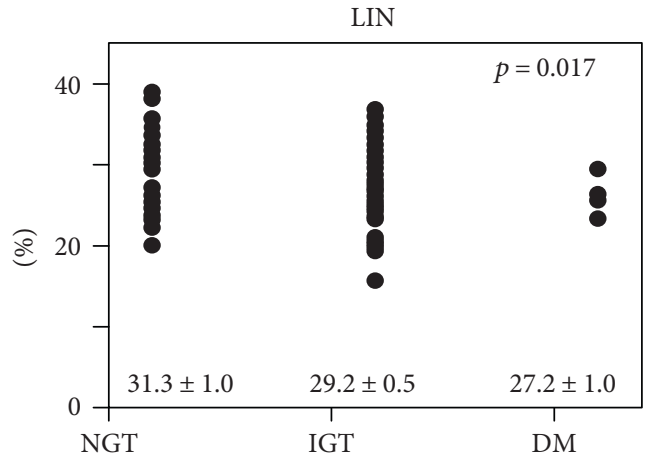

(a)

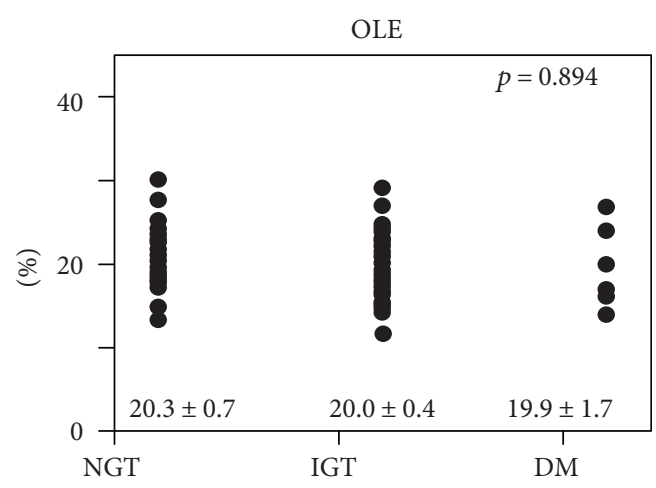

(c)

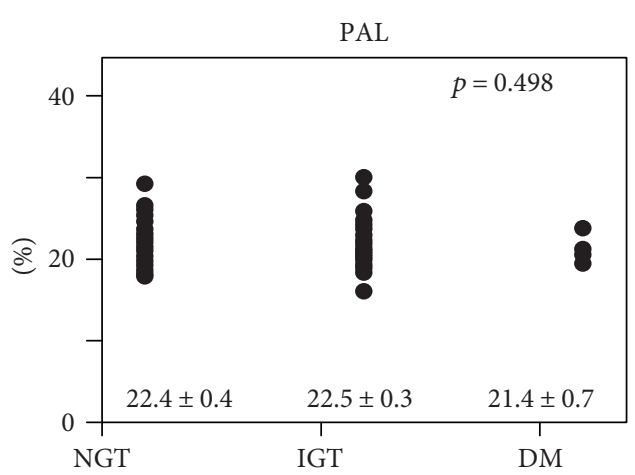

(b)

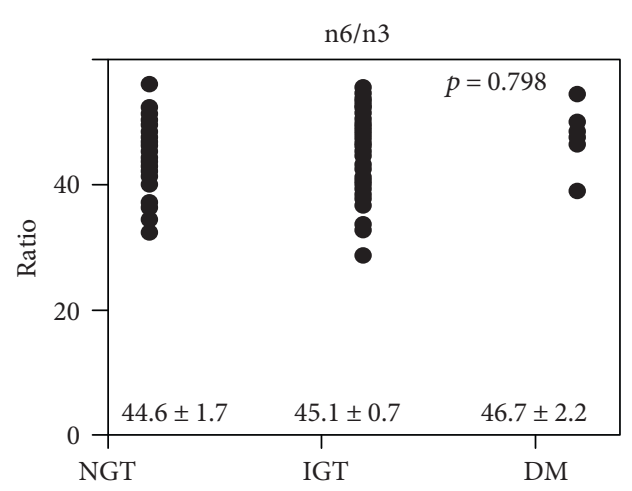

(d)

Figure 4: Percentages of linoleic acid (C18:2) (a), palmitic acid (C16:0) (b), and oleic acid (C18:1) (c) and n6/n3 PUFA ratio (d) in serum in normal glucose tolerant (NGT, $n=33$ ), impaired glucose tolerant (IGT, $n=71$ ), and diabetic (DM, $n=7)$ Japanese subjects by a cross-sectional evaluation. Kruskal-Wallis test was performed. Data are mean \pm SEM.

relationships between the major three FFAs and classification by OGTT. The percentage of linoleic acid in diabetic subjects was significantly lower than that in normal subjects (Figure 4(a)). On the other hand, the percentages of palmitic acid (Figure 4(b)) and oleic acid (Figure 4(c)) showed no significant differences among the groups. The n6/n3 PUFA ratio was not significantly different among the groups (Figure $4(\mathrm{~d})$ ).

Linoleic acid was significantly negatively correlated with visceral fat area $(n=30, r=-0.556, p=0.0014)$ (Figure 5(a)) and HOMA-IR $(n=31, r=-0.704, p<0.0001)$ (Figure 5(b)) by simple correlation analysis in normal subjects. In IGT subjects, linoleic acid was also significantly negatively correlated with visceral fat area $(n=66, r=-0.444, p<0.0001)$ (Figure 5(c)) and HOMA-IR $(n=69, r=-0.393, p=0.0008)$ (Figure 5(d)), as well as in normal subjects. The percentage of linoleic acid in IGT subjects with insulin resistance, which was defined as HOMA-IR > 2.5, was significantly lower than that in normal subjects (linoleic acid (\%); 31.32 \pm 0.97 in NGT $(n=30)$ versus $27.58 \pm 0.76$ in IGT with HOMAIR $>2.5(n=30), p=0.0036)$. In addition, the percentages of linoleic acid in IGT subjects with HOMA-IR $>2.5$ were significantly lower than those in IGT subjects with HOMA-IR $<2.5$ (linoleic acid (\%); $30.45 \pm 0.93$ in IGT with HOMA-IR $<2.5$ $(n=41)$ versus $27.58 \pm 0.76$ in IGT with HOMA-IR $>2.5$ $(n=30), p=0.0051)$.

\section{Discussion}

To investigate which FFA fraction is beneficial or deleterious for the development of type 2 diabetes in a race is important. In this study, we demonstrated that serum linoleic acid (\%) was negatively correlated with the accumulation of visceral fat in Japanese subjects by a cross-sectional evaluation. Linoleic acid, which is an essential fatty acid, is the greatest fatty acid component in serum in Japanese subjects. The percentage of linoleic acid was negatively correlated with visceral fat area and was significantly lower in diabetic subjects than in normal subjects in our study.

This observation in our study is consistent with a report that compared the risk of developing type 2 diabetes in relation to FFA fractions in serum in a longitudinal observation of Finnish subjects [17]. In addition, the percentage of linoleic acid was negatively correlated with visceral fat area in our study. Because the accumulation of visceral fat worsens glucose and lipid metabolism and is important in the development of type 2 diabetes [4], a low level of linoleic acid in serum might be related to the development of type 2 diabetes. However, our cross-sectional evaluation in humans raises concern that the causal relation between linoleic acid and visceral fat remains unknown.

It is reported that dietary safflower oil, which is rich in the linoleic acid, reduced trunk adipose mass and increased total 


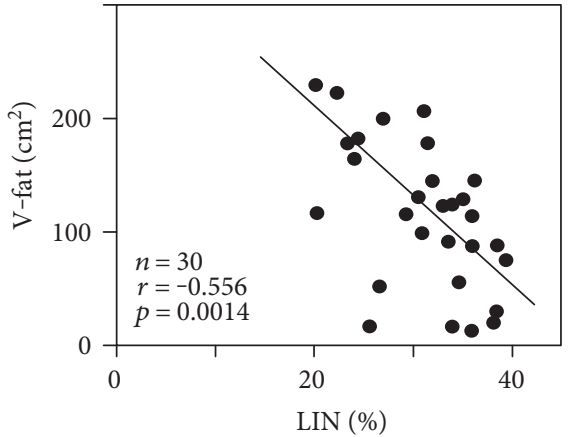

(a)

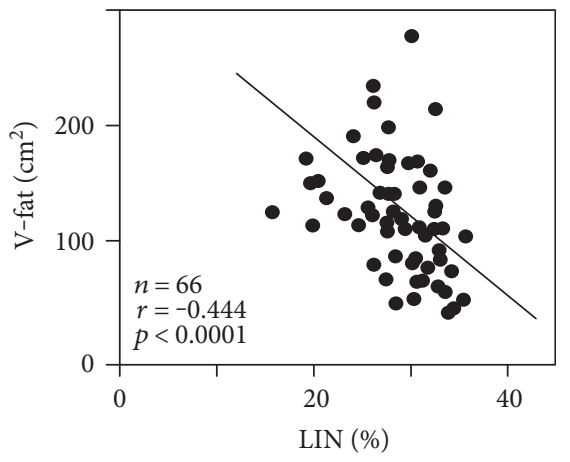

(c)

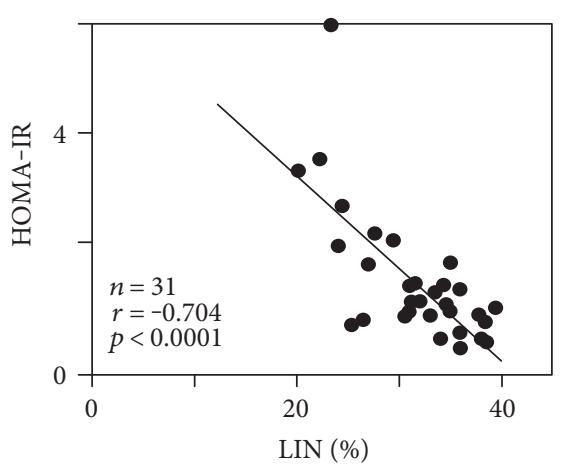

(b)

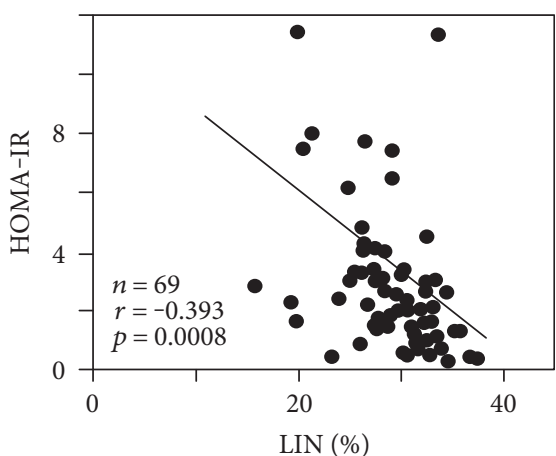

(d)

Figure 5: Relationships between ratio of serum linoleic acid (LIN, C18:2) and that of visceral fat area (V-fat) (a) and that of homeostasis model assessment insulin resistance index (HOMA-IR) (b) in subjects with normal glucose tolerance and ratio of serum linoleic acid and that of V-fat (c) and that of HOMA-IR (d) in subjects with impaired glucose tolerance, by simple correlation analysis.

body lean mass in obese women with type 2 diabetes and was associated with gene expression of uncoupling protein (UCP) 1 and UCP content in the adipose tissue of rats [18, 19]. Park et al. [20] showed that a diet supplemented with conjugated linoleic acid decreased body weight in mice and enhanced fatty acid $\beta$-oxidation, supported by increased carnitine palmitoyltransferase activity in the skeletal muscle and fat pad, and also that linoleic acid reduced lipoprotein lipase activity while apparently enhancing lipolysis in a study of 3T3-L1 adipocytes treated with conjugated linoleic acid. In the present study, including subjects without diabetes, the percentage of linoleic acid was significantly negatively correlated with visceral fat area. Although we cannot exclude that the reduced body weight by linoleic acid in an animal study was due to a toxic effect on adipocytes, it is rational that visceral fat does not cause a decrease in linoleic acid level in serum, but linoleic acid decreases visceral fat in humans.

Adiponectin is generally present in plasma at a high concentration and is inversely associated with visceral fat accumulation [21]. High molecular weight form of adiponectin is considered an active form of adiponectin in vitro $[22,23]$. Clinical studies $[24,25]$ also suggest that HMW adiponectin is more useful as an indicator than is total adiponectin, particularly in type 2 diabetic patients. We also conducted a cross-sectional study in healthy Japanese male subjects without any medication and reported that HMW adiponectin measured by ELISA was as effective as the $\mathrm{HMW} /$ total adiponectin ratio for predicting insulin resistance and/or metabolic syndrome [26]. Serum linoleic acid was correlated with HMW adiponectin in our study. It is suggested that linoleic acid and linoleic acid-derived fatty acids might increase serum adiponectin level through Keto A, which is a linoleic acid-derived fatty acid produced by gut lactic acid bacteria, and induce adipocyte differentiation via the activation of peroxisome proliferator-activated receptor $\gamma(\operatorname{PPAR}-\gamma)[27,28]$. This mechanism might be associated with the finding that pioglitazone, a PPAR- $\gamma$ agonist, induced an increase in serum adiponectin level in humans [29].

Palmitic acid, which is the second major component in Japanese subjects, was correlated with BMI and ALT (Figure 2). Previously, we have shown that a palmitic acid-supplemented diet might cause deterioration of glucose tolerance by suppression of insulin secretion from pancreatic $\beta$ cells in mice [8]. However, there was no difference in the level of palmitic acid among the normal, IGT, and diabetic groups in the current human study (Figure 4). It is reported that a palmitic acid-rich diet might change the 
gut microbiota and induce weight gain and hepatic lipid accumulation compared to an unsaturated fat diet in mice [30-32]. The discrepancies in the reported findings might be due to species differences.

Oleic acid, which is the third major FFA component in Japanese subjects, was correlated with serum TG and negatively correlated with HDL-C (Figure 3), while it was not correlated with parameters associated with insulin. In addition, there was no difference in oleic acid level among the normal, IGT, and diabetic groups (Figure 4). It is widely regarded that a Mediterranean diet, which is rich in oleic acid (olive oil), might be beneficial for the prevention and treatment of type 2 diabetes $[33,34]$. On the other hand, in a recent study, oleic acid plasma level was shown to be a selective biomarker of impaired glucose tolerance in several cohorts [35]. These discrepancies might be related to racial differences. Investigation of the appropriate oleic acid intake in different races might be important.

The n6/n3 PUFA ratio is suggested to be important in disease development, for example, cardiovascular disease in rodent models fed with a high-fat diet [36]. However, the relationships of n6/n3 PUFA ratio and type 2 diabetes are not clear. In the present study, n6/n3 PUFA ratio was not significantly different among the normal, IGT, and diabetes groups (Figure $4(\mathrm{~d})$ ).

This study has several limitations. First, the sample size was small. Second, the study design did not allow for correlations between the dietary components of FFAs and the serum components of FFAs. Third, this cross-sectional study design raises concern that the causal relation between fatty acids and parameters remains unknown, as we discussed above. Further longitudinal studies are needed with a greater number of subjects. Lastly, the precise mechanisms for the results in this cross-sectional study should be elucidated. Recently, several studies have suggested that differences in the dietary composition might change the gut microbiota in obesity and type 2 diabetes [37-39]. de Wit et al. [32] showed that a diet rich in unsaturated fat induced changes in gut microbiota composition and mucosal PPAR $\alpha$ target gene expression. Dietary trans-10, cis-12-conjugated linoleic acid ( $t 10 c 12$ CLA) significantly decreased visceral fat mass in mice. Analysis of the microbiota composition under $t 10 c 12$-CLA supplementation revealed a lower proportion of Firmicutes and a higher proportion of Bacteroidetes compared with that under no supplementation [40]. Linoleic acid might change the gut microbiota composition and reduce visceral fat.

\section{Conclusion}

In conclusion, we demonstrated that linoleic acid percentage was negatively correlated with the accumulation of visceral fat in relation to a reduction of insulin resistance in Japanese subjects.

\section{Disclosure}

The authors alone are responsible for the content and writing of this article. An earlier version of this work was presented as an abstract at the 71st Annual Meeting of the American Diabetes Association, 2011.

\section{Conflicts of Interest}

The authors report no conflict of interest.

\section{Acknowledgments}

This work was supported by JSPS KAKENHI, Grant nos. 23500854 and 26350905. The authors thank Dr. Wendy Gray for editing the paper.

\section{References}

[1] International Diabetes Federation, Diabetes Atlas 7th edition, International Diabetes Federation, Brussels, Belgium, 2015.

[2] P. Hossain, B. Kawar, and M. El Nahas, "Obesity and diabetes in the developing world - a growing challenge," The New England Journal of Medicine, vol. 356, no. 3, pp. 213-215, 2007.

[3] S. M. Haffner, S. Lehto, T. Rënnemaa, K. Pyërala, and M. Laakso, "Mortality from coronary heart disease in subjects with type 2 diabetes and in nondiabetic subjects with and without prior myocardial infarction," The New England Journal of Medicine, vol. 339, no. 4, pp. 229-234, 1998.

[4] S. Fujioka, Y. Matsuzawa, K. Tokunaga, and S. Tarui, "Contribution of intraabdominal fat accumulation to the impairment of glucose and lipid metabolism in human obesity," Metabolism, vol. 36, no. 1, pp. 54-59, 1987.

[5] H. Hirose, Y. H. Lee, L. R. Inman, Y. Nagasawa, J. H. Johnson, and R. H. Unger, "Defective fatty acid-mediated $\beta$-cell compensation in Zucker diabetic fatty rats," The Journal of Biological Chemistry, vol. 271, no. 10, pp. 5633-5637, 1996.

[6] G. Boden, "Role of fatty acids in the pathogenesis of insulin resistance and NIDDM," Diabetes, vol. 46, no. 1, pp. 3-10, 1997.

[7] T. Kawai, H. Hirose, Y. Seto, H. Fujita, and T. Saruta, "Chronic effects of different fatty acids and leptin in INS-1 cells," Diabetes Research and Clinical Practice, vol. 51, no. 1, pp. 1-8, 2001.

[8] T. Hirata, T. Kawai, H. Hirose et al., "Palmitic acid-rich diet suppresses glucose-stimulated insulin secretion (GSIS) and induces endoplasmic reticulum (ER) stress in pancreatic islets in mice," Endocrine Research, vol. 41, no. 1, pp. 8-15, 2016.

[9] S. M. Lopes, S. L. Trimbo, E. A. Mascioli, and F. L. Blackburn, "Human plasma fatty acid variations and how they are related to dietary intake," The American Journal of Clinical Nutrition, vol. 53, no. 3, pp. 628-637, 1991.

[10] E. J. Feskens, S. M. Virtanen, L. Räsänen et al., "Dietary factors determining diabetes and impaired glucose tolerance. A 20-year follow-up of the Finnish and Dutch cohorts of the Seven Countries Study," Diabetes Care, vol. 18, no. 8, pp. 1104-1112, 1995.

[11] S. G. Anderson, T. A. B. Sanders, and J. K. Cruickshank, "Plasma fatty acid composition as a predictor of arterial stiffness and mortality," Hypertension, vol. 53, no. 5, pp. 839845, 2009.

[12] K. Li, B. A. McNulty, A. M. Tiernery et al., "Dietary fat intakes in Irish adults in 2011: how much has changed in 10 years?" British Journal of Nutrition, vol. 115, no. 10, pp. 1798-1809, 2016. 
[13] K. G. M. M. Alberti and P. Z. Zimmet, "Definition, diagnosis and classification of diabetes mellitus and its complications. Provisional report of a WHO consultation," Diabetic Medicine, vol. 15, no. 7, pp. 539-553, 1998.

[14] M. Haug, I. Dieterich, C. Laubach, D. Reinhardt, and G. Harzer, "Capillary gas chromatography of fatty acid methyl esters from human milk lipid subclasses," Journal of Chromatography, vol. 279, pp. 549-553, 1983.

[15] D. R. Matthews, J. P. Hosker, A. S. Rudenski, B. A. Naylor, D. F. Treacher, and R. C. Turner, "Homeostasis model assessments: insulin resistance and $\beta$-cell function from fasting plasma glucose and insulin concentrations in man," Diabetologia, vol. 28, no. 7, pp. 412-419, 1985.

[16] T. Kawai, I. Takei, Y. Oguma et al., "Effects of troglitazone on fat distribution in the treatment of male type 2 diabetes," Metabolism, vol. 48, no. 9, pp. 1102-1107, 1999.

[17] B. Vessby, A. Aro, E. Skarfors, L. Berqlund, I. Salminen, and H. Lithell, "The risk to develop NIDDM is related to the fatty acid composition of the serum cholesterol esters," Diabetes, vol. 43, no. 11, pp. 1353-1357, 1994.

[18] T. Matsuo, H. Takeuchi, H. Suzuki, and M. Suzuki, "Body fat accumulation is greater in rats fed a beef tallow diet than in rats fed a safflower or soybean oil diet," Asia Pacific Journal of Clinical Nutrition, vol. 11, no. 4, pp. 302-308, 2002.

[19] L. E. Norris, A. L. Collene, M. L. Asp et al., "Comparison of dietary conjugated linoleic acid with safflower oil on body composition in obese postmenopausal woman with type 2 diabetes mellitus," The American Journal of Clinical Nutrition, vol. 90, no. 3, pp. 468-476, 2009.

[20] Y. Park, K. J. Albright, W. Liu, J. M. Storkson, M. E. Cook, and M. W. Pariza, "Effect of conjugated linoleic acid on body composition in mice," Lipids, vol. 32, no. 8, pp. 853-858, 1997.

[21] Y. Matsuzawa, "Establishment of a concept of visceral fat syndrome and discovery of adiponectin," Proceedings of the Japan Academy. Series B, Physical and Biological Sciences, vol. 86, no. 2, pp. 131-141, 2010.

[22] N. Ouchi, H. Kobayashi, S. Kihara et al., "Adiponectin stimulates angiogenesis by promoting cross-talk between AMP-activated protein kinase and Akt signaling in endothelial cells," The Journal of Biological Chemistry, vol. 279, no. 2, pp. 1304-1309, 2004.

[23] Y. Wang, K. S. Lam, J. Y. Xu et al., “Adiponectin inhibits cell proliferation by interacting with several growth factors in an oligomerization-dependent manner," The Journal of Biological Chemistry, vol. 280, no. 18, pp. 18341-18347, 2005.

[24] H. Kobayashi, N. Ouchi, S. Kihara et al., "Selective suppression of endothelial cell apoptosis by the high molecular weight form of adiponectin," Circulation Research, vol. 94, no. 4, pp. e27-e31, 2004.

[25] U. B. Pajvani, M. Hawkins, T. P. Combs et al., "Complex distribution, not absolute amount of adiponectin, correlates with thiazolidinedione-mediated improvement in insulin sensitivity," The Journal of Biological Chemistry, vol. 279, no. 13, pp. 12152-12162, 2004.

[26] Y. Seino, H. Hirose, I. Saito, and H. Itoh, "High molecular weight multimer form of adiponectin as a useful marker to evaluate insulin resistance and metabolic syndrome in Japanese men," Metabolism, vol. 56, no. 11, pp. 1493-1499, 2007.

[27] S. A. Kliewer, S. S. Sundseth, S. A. Jones et al., "Fatty acids and eicosanoids regulate gene expression through direct interactions with peroxisome proliferator-activated receptor alpha and gamma," Proceedings of the National Academy of Sciences of the United States of America, vol. 94, no. 9, pp. 4318-4323, 1997.

[28] T. Goto, Y. Kim, T. Furuzono et al., "10-oxo-12(Z)-octadecenoic acid, a linoleic acid metabolite produced by gut lactic acid bacteria, potently activates PPAR $\gamma$ and stimulates adipogenesis," Biochemical and Biophysical Research Communications, vol. 459, no. 4, pp. 597-603, 2015.

[29] H. Hirose, T. Kawai, Y. Yamamoto et al., "Effects of pioglitazone on metabolic parameters, body fat distribution, and serum adiponectin levels in Japanese male patients with type 2 diabetes," Metabolism, vol. 51, no. 3, pp. 314-317, 2002.

[30] R. E. Ley, F. Backhed, P. Turnbaugh, C. A. Lozaupone, R. D. Knight, and J. I. Gordon, "Obesity alters gut microbial ecology," Proceedings of the National Academy of Sciences of the United States of America, vol. 102, no. 31, pp. 11070-11075, 2005.

[31] G. Musso, R. Gambino, and M. Cassader, "Interactions between gut microbiota and host metabolism predisposing to obesity and diabetes," Annual Review of Medicine, vol. 62, pp. 361-380, 2011.

[32] N. de Wit, M. Derrien, H. Bosch-Vermeulen et al., "Saturated fat simulates obesity and hepatic steatosis and affects gut microbiota composition by an enhanced overflow of dietary fat to the distal intestine," American Journal of Physiology. Gastrointestinal and Liver Physiology, vol. 303, no. 5, pp. G589-G599, 2012.

[33] K. Esposito, M. I. Maiorino, A. Ceriello, and D. Giugliano, "Prevention and control of type 2 diabetes by Mediterranean diet: a systematic review," Diabetes Research and Clinical Practice, vol. 89, no. 2, pp. 97-102, 2010.

[34] D. Romaguera, M. Guevara, T. Norat et al., "Mediterranean diet and type 2 diabetes risk in the European Prospective Investigation into Cancer and Nutrition (EPIC) study: the InterAct project," Diabetes Care, vol. 34, no. 9, pp. 19131918, 2011.

[35] J. Cobb, A. Eckhart, A. Motsinger-Reif, B. Carr, L. Groop, and E. Ferrannini, " $\alpha$-Hydroxybutyric acid is a selective metabolite biomarker of impaired glucose tolerance," Diabetes Care, vol. 39, no. 6, pp. 988-995, 2016.

[36] H. Poudyal, S. K. Panchal, J. Waanders, L. Ward, and L. Brown, "Lipid redistribution by alpha-linoleic acid-rich chia seed inhibits stearoyl-CoA desaturase-1 and induces cardiac and hepatic protection in diet-induced obese rats," The Journal of Nutritional Biochemistry, vol. 23, no. 2, pp. 153-162, 2012.

[37] P. J. Turnbaugh, R. E. Ley, M. A. Mahowald, V. Magrini, E. R. Mardis, and J. I. Gordon, "An obesity-associated gut microbiome with increased capacity for energy harvest," Nature, vol. 444, no. 7122, pp. 1027-1031, 2006.

[38] P. Kovatcheva-Datchary and T. Arora, "Nutrition, the gut microbiome and the metabolic syndrome," Best Practice and Research. Clinical Gastroenterology, vol. 27, no. 1, pp. 59-72, 2013.

[39] S. M. Harakeh, I. Khan, T. Kumosani et al., "Gut microbiota: a contributing factor to obesity," Frontiers in Cellular and Infection Microbiology, vol. 6, article 95, pp. 1-11, 2016.

[40] T. M. Marques, R. Wall, O. O'Sullivan et al., "Dietary trans-10, cis-12-conjugated linoleic acid alters fatty acid metabolism and microbiota composition in mice," British Journal of Nutrition, vol. 113, no. 5, pp. 728-738, 2015. 


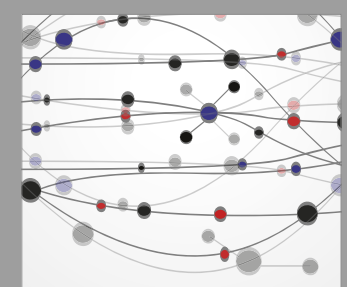

The Scientific World Journal
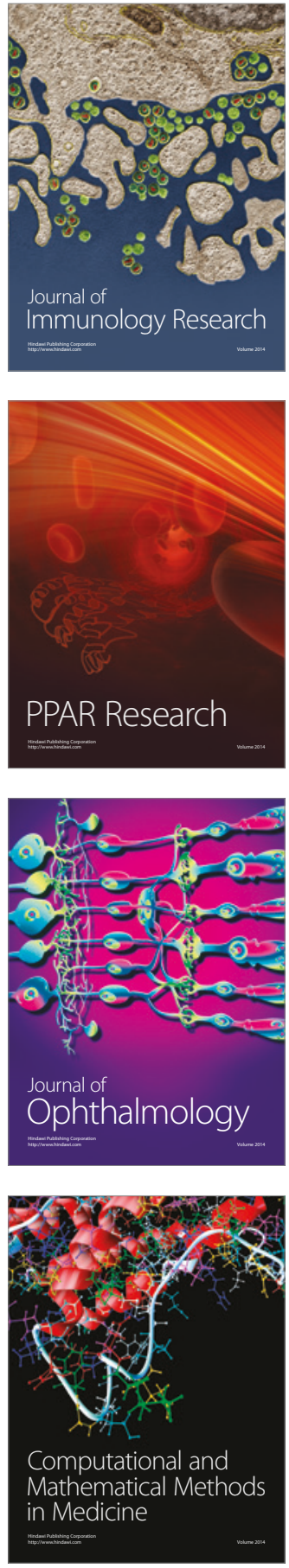

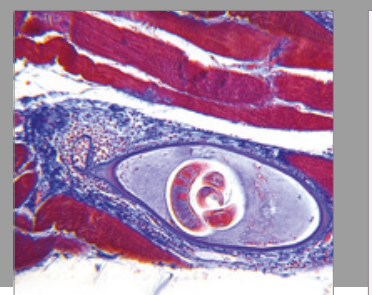

Gastroenterology Research and Practice
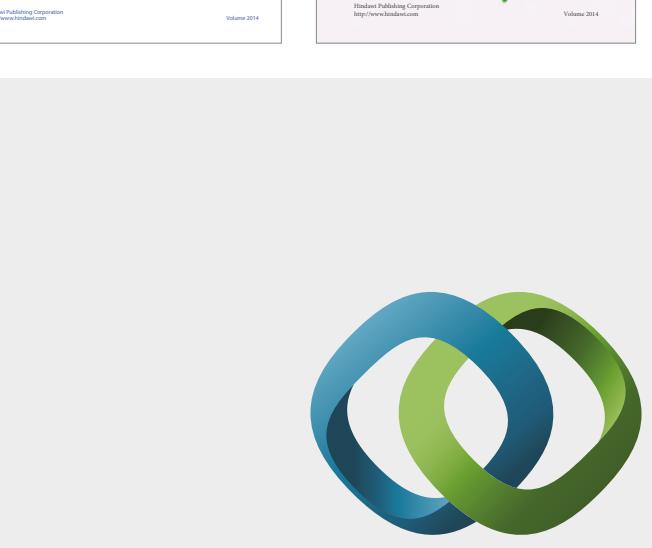

\section{Hindawi}

Submit your manuscripts at

https://www.hindawi.com
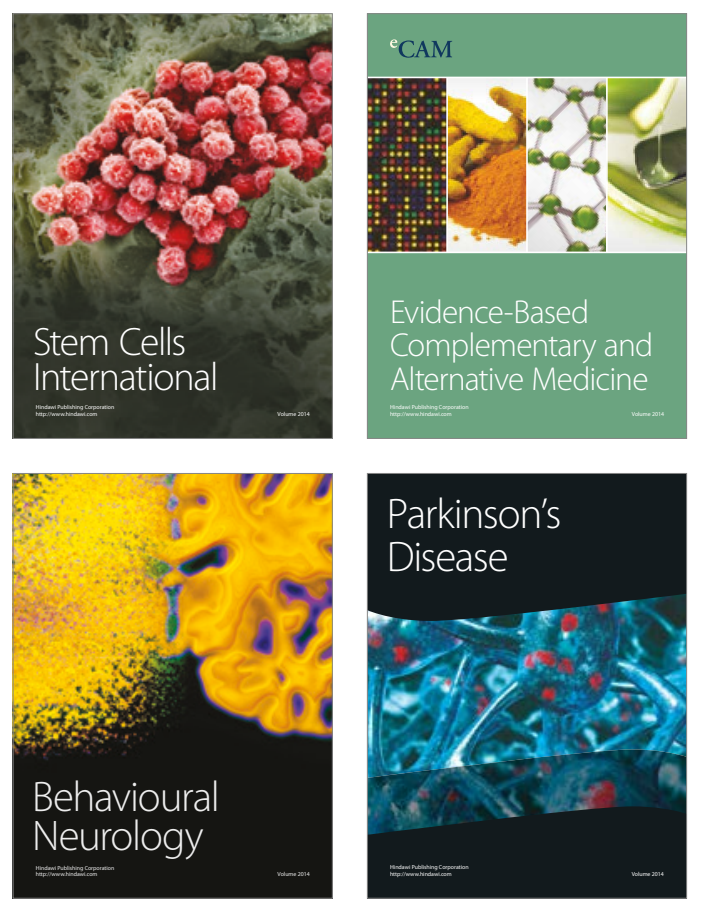
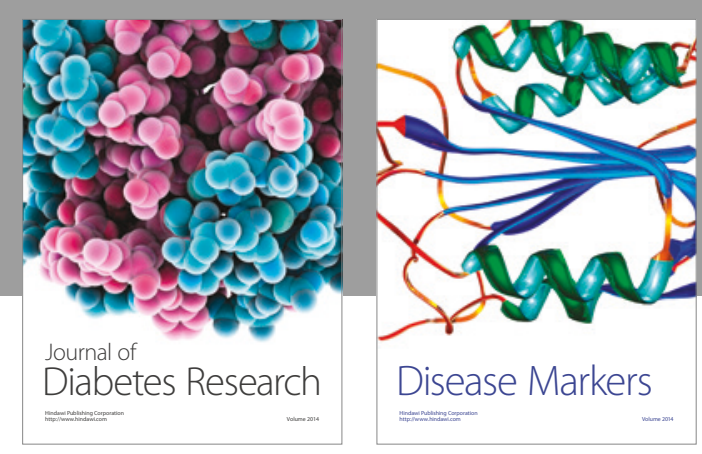

Disease Markers
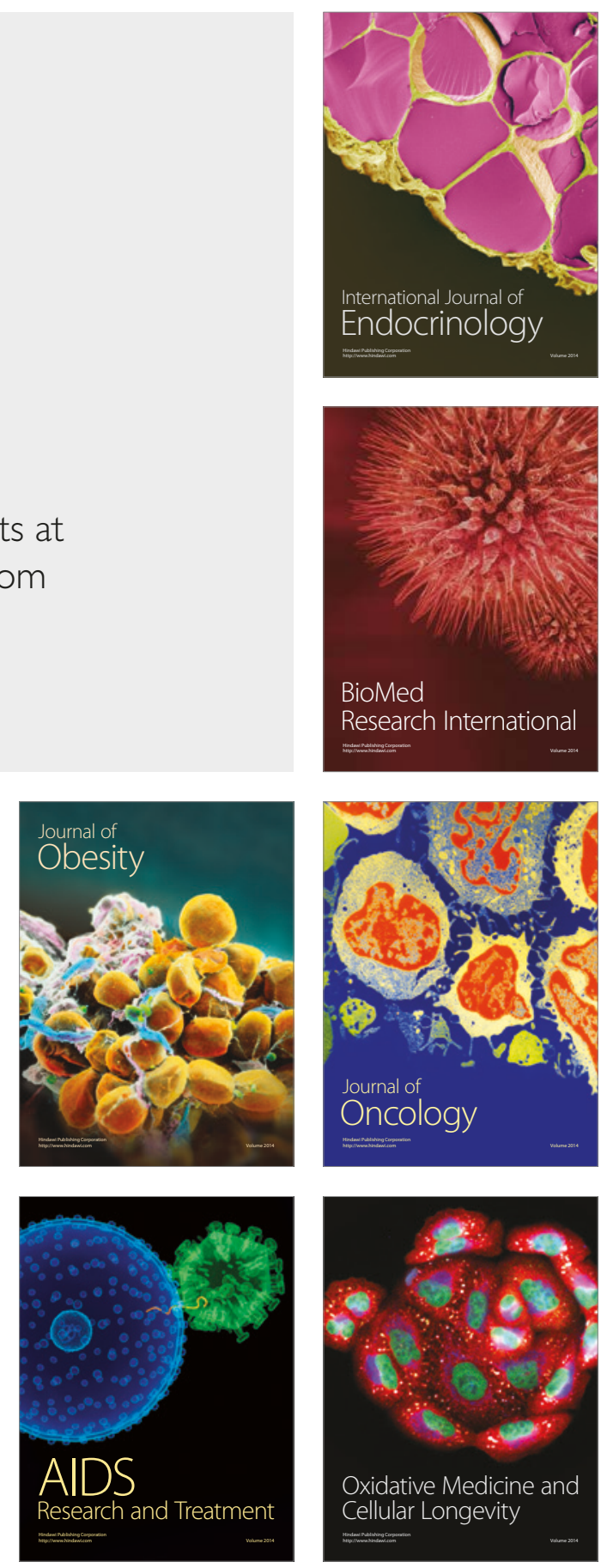